\title{
Effects of alprostadil combined with hyperbaric oxygen on hearing recovery and hemorheology in patients with sudden sensorineural hearing loss and analysis of related influencing factors
}

\author{
TAO FENG, QINQIN ZHANG, JIASHA WEI, XIAO WANG and YANG GENG \\ Department of Otolaryngology, Zibo Central Hospital, Zibo, Shandong 255036, P.R. China
}

Received August 27, 2019; Accepted September 8, 2021

DOI: $10.3892 /$ etm.2022.11167

\begin{abstract}
The causes of sudden sensorineural hearing loss (SSNHL) are unclear, and the condition cannot be treated with specific regimens. The present study was designed to explore the effects of alprostadil combined with hyperbaric oxygen (HBO) on hearing recovery and hemorheology in patients with SSNHL, and to analyze factors influencing treatment efficacy. in total, 104 patients with SSNHL were enrolled between January 2015 and July 2018 in a randomized controlled trial. Group A $(n=52)$ received alprostadil treatment and Group B $(n=52)$ were treated with HBO plus alprostadil. Following treatment, the overall recovery rates of tinnitus, aural fullness and vertigo in Group B were significantly higher than those in Group $\mathrm{A}(\mathrm{P}<0.05)$. Low-, middle- and high-shear whole blood viscosity and whole blood viscosity in the two groups significantly decreased, and those in Group B were significantly lower than those in Group A $(\mathrm{P}<0.05)$. The present results indicated that the combination of alprostadil with $\mathrm{HBO}$ can improve the therapeutic effect on SSNHL, reduce blood viscosity and improve coagulation function, thus significantly promoting hearing recovery. Further, SSNHL treatment efficacy was influenced by age, time of onset, hearing threshold, presence or absence of tinnitus, plasma viscosity and serum $\mathrm{C}$-reactive protein levels. The trial registration number for the present study was: NCT02563872.
\end{abstract}

\section{Introduction}

Nowdays, more and more people (particularly the young) suffer from sudden sensorineural hearing loss (SSNHL) $(1,2)$. The disease has unknown causes and is characterized by continuous decline of two or more hearing frequencies, rapid progression and short time of onset $(3,4)$. A previous study

Correspondence to: Dr Yang Geng, Department of Otolaryngology, Zibo Central Hospital, 54 Gongqing Tuan Road, Zhangdian, Zibo, Shandong 255036, P.R. China

E-mail: yanggengyx@163.com

Key words: hyperbaric oxygen, combination, alprostadil, sudden sensorineural hearing loss, hearing, hemorheology, influencing factor has shown that most patients with SSNHL experience hearing loss accompanied by vestibular trauma, which seriously damages hearing and significantly affects prognoses (5). The factors causing SSNHL are unclear, and the disease cannot be treated with specific regimens. The current standard treatment for sudden hearing loss is a tapered course of oral high-dose corticosteroids to increase circulation to the inner ear (6). In the present study, alprostadil and the combination of alprostadil and $\mathrm{HBO}$ were investigated as treatments for SSNHL.

Alprostadil regulates the activities of adenylate cyclase and phosphodiesterase in vascular smooth muscle cells, increases the cellular concentration of cyclic adenosine monophosphate and significantly relaxes vascular smooth muscles. It also reduces platelet activity and blood viscosity to inhibit blood aggregation, and eliminates oxygen free radicals, thus improving vascular function in inner ear microcirculation disorders and promoting blood circulation in the ears $(7,8)$. As a naturopathy that fuses oxygen and blood through high-concentration air pressure in a chamber, hyperbaric oxygen (HBO) improves tissue hypoxia and inner ear circulation disorders in the supportive treatment of SSNHL $(9,10)$. A previous study reported that, while medicines alone are limited in treating the disease, a combination of medicines with HBO increases partial pressure of blood oxygen in the body and improves inner ear ischemia, hypoxia and edema with positive therapeutic effects (11). Currently, SSNHL is commonly treated with alprostadil and HBO, and the value of this treatment has gradually increased in recent years.

In the present study, the therapeutic value of alprostadil combined with HBO are discussed in detail, and the effects of this combination on hearing recovery and hemorheology are assessed. The possible influencing factors of treatment efficacy for SSNHL are comprehensively analyzed to improve the overall therapeutic effect.

\section{Materials and methods}

General information. A total of 104 patients with SSNHL admitted from January 2015 to July 2018 were enrolled in the randomized controlled trial. Group A $(n=52)$ received alprostadil treatment and Group $B(n=52)$ was treated with HBO plus alprostadil. Of the total patients, 55 and 49 were males and females, respectively, with a mean age of 
44.62 \pm 11.45 years (age range, 20-59 years) and average body mass index (BMI) of $21.63 \pm 3.35 \mathrm{~kg} / \mathrm{m}^{2}$. The time of onset ranged from 2 to 14 days with an average of $8.34 \pm 4.62$ days. The patients experienced flat descending SSNHL $(n=26)$, low-frequency descending SSNHL $(n=33)$ and high-frequency descending SSNHL $(n=45)$.

Inclusion and exclusion criteria. The inclusion criteria were as follows: i) Patients with hearing loss of $>30 \mathrm{~dB}$, affecting three or more frequencies and arising over $<3$ days; ii) patients with unilateral deafness accompanied by vertigo, vestibular dysfunction, aural fullness, tinnitus and with normal cranial nerve examination results; and iii) patients not allergic to alprostadil.

The exclusion criteria were as follows: i) Patients who had recently taken ear medicines or undergone surgical treatment; ii) patients with other pathological deafness; iii) patients with ear trauma and critical strikes; iv) patients with cognitive or communication disorders; and v) patients with poor compliance. All patients and their families agreed to participate in this study and signed an informed consent form. The present study was approved by the Medical Ethics Committee of Zibo Central Hospital (Zibo, China).

Experimental reagents and materials. Alprostadil was purchased from Hagaoke White Swan Pharmaceutical Group Co., Ltd. (State Food and Drug Administration; approval no. H23023072). A HBO chamber was purchased from Ningbo KQD Plastic Tech Co., Ltd. A non-invasive hemorheometer was purchased from Beckman Coulter, Inc. A fully automated coagulation analyzer was purchased from Jiangsu Horner Medical Instrument Co., Ltd.

Experimental methods. Patients in Group A were treated with alprostadil. Alprostadil $(10 \mu \mathrm{g})$ was dissolved and diluted in $0.9 \% \mathrm{NaCl}$ solution $(200 \mathrm{ml})$ and administered via intravenous drip. The standard scheme was once per day, and one course of treatment was completed in 10 days. The patients were treated for two courses and the interval between courses was 3 days.

Patients in Group B were treated with HBO combined with alprostadil. The pressure of the HBO chamber was adjusted to $0.2 \mathrm{MPa}$ and then boosted for $20 \mathrm{~min}$. The patients inhaled pure oxygen for $1 \mathrm{~h}$. The standard scheme was performed once per day, and one course of treatment was completed in 10 days. The patients were treated for two courses and the interval between courses was 3 days.

Outcome measures. The outcomes measured were as follows: i) Total effective rate of treatment: The therapeutic effect was divided into cured (symptoms completely disappeared), markedly effective (symptoms basically disappeared and hearing threshold increased by $30 \mathrm{~dB}$ ), effective (symptoms were significantly relieved and hearing threshold increased by 15-30 dB) and ineffective (symptoms were still significant and hearing threshold increased by $<15 \mathrm{~dB}$ ). The total effective rate of treatment $=($ curative + markedly effective + effective cases)/total number of cases $x 100 \%$ (12); ii) hearing recovery: Improvements of tinnitus, aural fullness and vertigo in the two groups were observed by clinicians and recorded as disappearance, alleviation, or ineffectiveness. Hearing recovery rate $=($ disappearance + alleviation cases $) /$ total number of cases x100\% (13); iii) Hemorheological index changes: A non-invasive hemorheometer was used to detect and assess changes in low-, middle- and high-shear whole blood viscosity as well as whole blood viscosity before and after treatment; iv) Coagulation function: A fully automated coagulation analyzer was used to detect, record and assess changes in fibrinogen (FIB), platelet factor (PF), prothrombin time (PT) and activated partial thrombin time (APTT); and v) Analysis of related influencing factors: According to the efficacy of $\mathrm{HBO}$ combined with alprostadil, the patients were divided into effective and ineffective groups. Multivariate logistic regression was used to analyze the factors influencing the efficacy of treatment for SSNHL.

Statistical methods. SPSS 20.0 (IBM Corp.) was used for statistical analysis. Measurement data were analyzed by the $\chi^{2}$ test and expressed as the mean \pm SD. Multiple comparisons were performed using a mixed ANOVA followed by an appropriate post hoc test (Bonferroni's correction) in order to compare indicators before and after treatment. Multivariate logistic regression was used to analyze factors influencing the efficacy of treatment for SSNHL. Differences with $\mathrm{P}<0.05$ were statistically significant.

\section{Results}

Comparison of general information. No significant differences were observed between Group A and B with regards to sex, age, BMI and time or site of onset ( $P>0.05$; Table I).

Comparison of total effective rate of treatment. The total effective rate of treatment in Group A was lower compared with that in Group B $(\mathrm{P}<0.05$; Table II).

Comparison of hearing recovery. Tinnitus, ear tightness and vertigo or vestibular dysfunction improved in the two groups following treatment. However, after treatment, the overall recovery rate of hearing in Group B was higher than that in Group A ( $\mathrm{P}<0.05$; Table III).

Comparison of hemorheological index changes. Before treatment, there were no significant differences between Group A and $\mathrm{B}$ in hemorheological indices $(\mathrm{P}>0.05)$. After treatment, low-, middle- and high-shear whole blood viscosity decreased in the two groups, and these hemorheological indices in Group B were lower than those in Group A ( $\mathrm{P}<0.05$; Fig. 1).

Comparison of coagulation function. Before treatment, no significant differences were noted between Group A and B in PF, FIB, PT and APTT $(\mathrm{P}>0.05)$. After treatment, PF and FIB levels in the two groups decreased $(\mathrm{P}<0.05)$, and $\mathrm{PT}$ and APTT were prolonged $(\mathrm{P}<0.05)$. The improvement of these coagulation function parameters in Group B were significantly greater than that in Group A ( $\mathrm{P}<0.05$; Fig. 2).

Univariate logistic regression analysis. According to the efficacy of HBO combined with alprostadil, patients were divided into effective $(n=45)$ and ineffective $(n=7)$ groups. Univariate analysis of the clinical data showed that there were significant 
Table I. Comparison of baseline characteristics between patient groups.

\begin{tabular}{lccc}
\hline Groups & Group A $(\mathrm{n}=52)$ & Group B $(\mathrm{n}=52)$ & $\chi^{2}$-value/t \\
\hline Sex & & & 0.347 \\
Male & $26(50.00)$ & $29(55.77)$ & 0.556 \\
Female & $26(50.00)$ & $23(44.23)$ & 0.352 \\
Age, years & $44.74 \pm 11.26$ & $43.95 \pm 11.64$ & 0.244 \\
BMI, kg/m ${ }^{2}$ & $21.57 \pm 3.45$ & $21.73 \pm 3.23$ & 0.706 \\
Time of onset, days & $8.87 \pm 3.37$ & $8.25 \pm 3.58$ & 0.040 \\
Site of onset & & & 0.726 \\
Left ear & $31(59.62)$ & $30(57.69)$ & 0.482 \\
Right ear & $21(40.38)$ & $68.25 \pm 8.01$ & 0.576 \\
Hearing threshold, dB & $67.35 \pm 7.93$ & & 0.206 \\
Classification of deafness & $12(23.08)$ & $14(26.92)$ & 0.566 \\
Flat descent & $17(32.69)$ & $16(30.77)$ & 0.902 \\
Low frequency descent & $23(44.23)$ & $22(42.31)$ & \\
High frequency descent & & & \\
\hline
\end{tabular}

Data was expressed as $[\mathrm{n}(\%)]$ or mean $\pm \mathrm{SD}$.

Table II. Comparison of total effective rate of treatment.

\begin{tabular}{lccc}
\hline Groups & Group A (n=52) & Group B (n=52) & $\chi^{2}$-value \\
\hline Curative & $12(23.08)$ & $18(34.62)$ & - \\
Markedly effective & $11(21.15)$ & $13(25.00)$ & - \\
Effective & $13(25.00)$ & $14(26.92)$ & - \\
Ineffective & $16(20.77)$ & $7(13.46)$ & - \\
Total effective rate of treatment & $36(69.23)$ & $45(86.54)$ & - \\
\hline
\end{tabular}

differences between the two groups in age, time of onset, hearing threshold, presence or absence of tinnitus, plasma viscosity and C-reactive protein (CRP) level. Changes in plasma viscosity have a huge impact on the degree of deafness $(\mathrm{P}<0.05)$ whereas gender, and differences in the site of onset, types of hearing curves, and presence or absence of vertigo do not (P>0.05; Table IV).

Multivariate logistic regression analysis. Multivariate logistic regression analysis was conducted using efficacy of $\mathrm{HBO}$ combined with alprostadil in treating SSNHL as a dependent variable, and differences between the groups identified in the univariate analysis as independent variables (Table V). The results show that age, time of onset, hearing threshold, presence or absence of tinnitus, plasma viscosity, and serum CRP level were the influencing factors of the efficacy of $\mathrm{HBO}$ combined with alprostadil for SSNHL $(\mathrm{P}<0.05$; Table VI).

\section{Discussion}

SSNHL is a disease with clinical symptoms including tinnitus and vertigo. Its specific pathogenesis remains unclear, although it may be related to viral infection, inner ear microcirculation disorders and eardrum rupture $(14,15)$.
Studies have shown that viral infection and hemodynamic changes in patients with the disease result in lacunar obstruction after edema and damaged functional structure of the cochlea due to insufficient blood oxygen supply. This results in hearing loss and destruction of the inner ear, which affects maintenance of body balance and transmission of sound information. Deafness and tinnitus occur in severe cases. Therefore, to treat SSNHL, blood oxygen supply in the inner ear should be restored to normal levels $(16,17)$. Alprostadil, with a high bioavailability, delivers prostaglandin E1 to the lesion site, thereby improving its therapeutic stability (18). $\mathrm{HBO}$ increases the partial pressure and content of blood oxygen in patients, increases oxygen diffusion, and improves tissue hypoxia and blood supply, facilitating hair cell repair (19). It also increases blood flow, improves the blood supply capacity of the vertebrobasilar artery in the inner ear and improves the endothelial permeability of inner ear capillaries. This results in greatly improved repair efficiency of hair cells and vestibular nerve fibers (20). Few studies have investigated the combined effects of alprostadil and $\mathrm{HBO}$ on hearing recovery and hemorheology in patients with SSNHL. Therefore, the present study explored the efficacy and influencing factors of combining alprostadil with $\mathrm{HBO}$ in treating SSNHL. 
Table III. Comparison of hearing recovery $[\mathrm{n}(\%)]$.

\begin{tabular}{lcccc}
\hline Groups & Group A (n=52) & Group B (n=52) & $\chi^{2}$-value & P-value \\
\hline Tinnitus & & & 4.981 & 0.026 \\
Disappearance & $24(46.15)$ & $26(50.00)$ & - & - \\
Improved & $19(36.54)$ & $24(46.15)$ & - & - \\
Ineffective & $9(17.31)$ & $2(3.85)$ & - & - \\
Overall recovery rate & $43(82.69)$ & $50(96.15)$ & - & - \\
Aural Fullness & & & 4.308 & - \\
Disappearance & $25(48.08)$ & $29(55.77)$ & - & - \\
Improved & $17(32.69)$ & $20(38.46)$ & - & - \\
Ineffective & $10(19.23)$ & $3(5.77)$ & - & - \\
Overall recovery rate & $42(80.77)$ & $49(94.23)$ & 4.727 & - \\
Vertigo & & & - & - \\
Disappearance & $22(42.31)$ & $26(50.00)$ & - & - \\
Improved & $18(34.62)$ & $22(42.31)$ & - & - \\
Ineffective & $12(23.08)$ & $48(7.69)$ & - \\
Overall recovery rate & $40(76.92)$ & & - \\
\hline
\end{tabular}

A

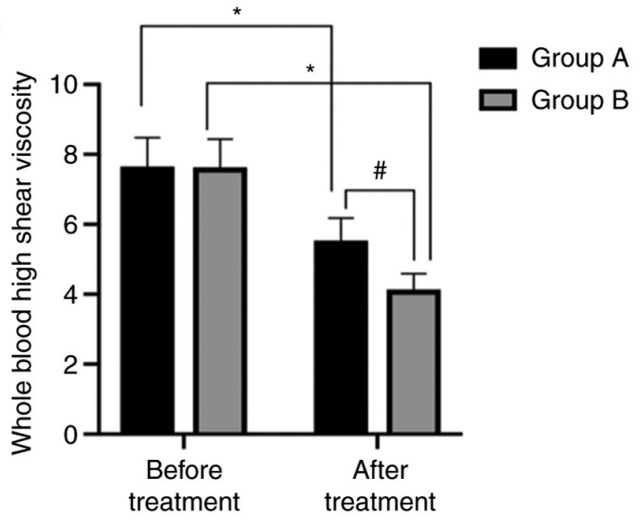

C

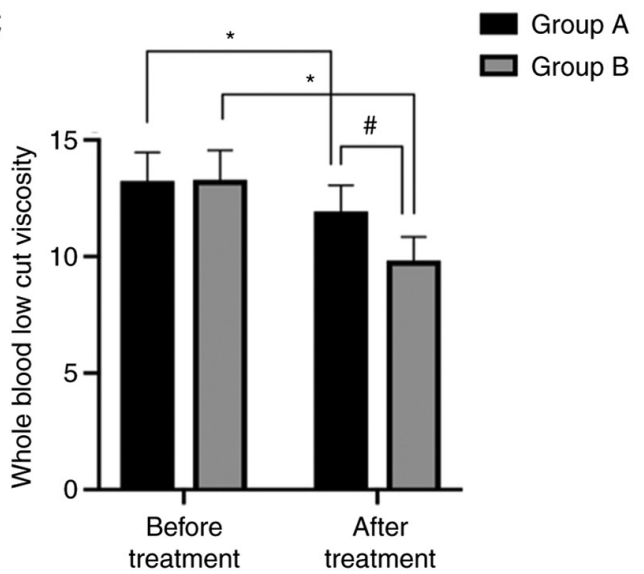

B

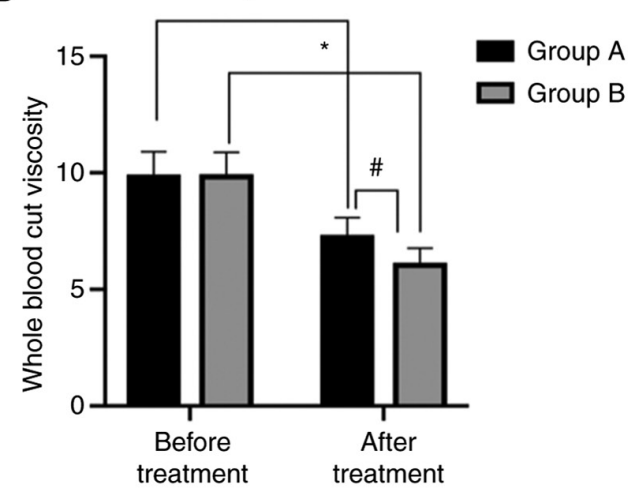

D

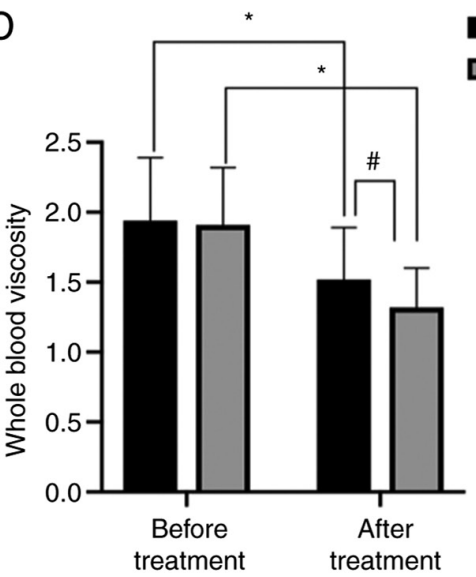

Group A

Group B

Figure 1. Comparison of hemorheological index changes. (A) After treatment, high-shear whole blood viscosity in both groups significantly decreased, and was significantly lower in Group B than in Group A. (B) After treatment, middle-shear whole blood viscosity in both groups significantly decreased, and was significantly lower in Group B than in Group A. (C) After treatment, low-shear whole blood viscosity in both groups significantly decreased, and was significantly lower in Group B than in Group A. (D) After treatment, whole blood viscosity in both groups significantly decreased, and was significantly lower in Group B than in Group A. ${ }^{*} \mathrm{P}<0.05$ vs. pre-treatment; ${ }^{*} \mathrm{P}<0.05$ vs. Group A.

In the current study, patients in Group B (treated with combined alprostadil and HBO) had: i) Higher total effective rate of treatment and overall recovery rate of hearing $(\mathrm{P}<0.05)$; ii) lower blood viscosity after treatment 
Table IV. Univariate logistic regression analysis.

\begin{tabular}{|c|c|c|c|c|}
\hline Groups & Effective group $(n=45)$ & Ineffective group $(n=7)$ & $\chi^{2}$-value/t & P-value \\
\hline Sex & & & 0.006 & 0.937 \\
\hline Male & $25(55.56)$ & $4(57.14)$ & & \\
\hline Female & $20(44.44)$ & $3(42.86)$ & & \\
\hline Age, years & $41.34 \pm 9.26$ & $49.93 \pm 10.25$ & 2.253 & 0.029 \\
\hline Time of onset, days & $7.51 \pm 3.25$ & $11.25 \pm 3.54$ & 2.148 & 0.037 \\
\hline Site of onset & & & 0.001 & 0.975 \\
\hline Left ear & $26(57.78)$ & $4(57.14)$ & & \\
\hline Right ear & $19(42.22)$ & $3(42.86)$ & & \\
\hline Hearing threshold, $\mathrm{dB}$ & $64.55 \pm 8.25$ & $73.44 \pm 8.41$ & 2.646 & 0.011 \\
\hline Types of hearing curves & & & 0.625 & 0.429 \\
\hline Low-frequency or flat descent & $20(44.44)$ & $2(28.57)$ & & \\
\hline High-frequency descent or total deafness & $25(55.56)$ & $5(71.43)$ & & \\
\hline Tinnitus & & & 4.127 & 0.042 \\
\hline Yes & $20(44.44)$ & $6(85.71)$ & & \\
\hline No & $25(55.56)$ & $1(14.29)$ & & \\
\hline Vertigo & & & 1.486 & 0.223 \\
\hline Yes & $21(46.67)$ & $5(71.43)$ & & \\
\hline No & $24(53.33)$ & $2(28.57)$ & & \\
\hline Whole blood viscosity, MPa.s & $1.24 \pm 0.21$ & $1.63 \pm 0.26$ & 4.431 & $<0.001$ \\
\hline Serum CRP level, $\mu \mathrm{mol} / 1$ & $21.23 \pm 3.57$ & $28.23 \pm 3.63$ & 4.816 & $<0.001$ \\
\hline
\end{tabular}

Table V. Variable assignment in logistic regression analysis.

Factors

Assignment

Age

Time of onset

Hearing threshold

Tinnitus

Plasma viscosity

Serum CRP level
A continuous variable analyzed by initial data A continuous variable analyzed by initial data A continuous variable analyzed by initial data Yes $=1$, no $=0$

A continuous variable analyzed by initial data A continuous variable analyzed by initial data
$(\mathrm{P}<0.05)$; and iii) greater improvement of coagulation function parameters after treatment $(\mathrm{P}<0.05)$ relative to those treated with alprostadil only (Group A). Previous studies have shown that alprostadil significantly relieves pathological symptoms and promotes hearing recovery in patients with SSNHL (21). The combination of alprostadil and HBO leads to improvement of peripheral vascular resistance in terms of antiplatelet aggregation by increasing the activity of intracellular cyclic adenosine monophosphate. Moreover, it accelerates the absorption of extravascular tissue fluid to correct local edema, ensures ear blood supply and reduces the effects of immune response damage on the body $(22,23)$. These results are consistent with the present findings, which suggest that a combination of alprostadil and HBO (with wider and more effective clinical value) can improve the therapeutic efficiency of alprostadil and promote hearing recovery.
Multivariate logistic regression analysis revealed that age, time of onset, hearing threshold, presence or absence of tinnitus, plasma viscosity and serum CRP level are factors that influence the efficacy of alprostadil and $\mathrm{HBO}$ in treating SSNHL $(\mathrm{P}<0.05)$. A similar study suggested that age growth combined with complications results in slow blood circulation and deterioration of hearing function, which may affect the onset of SSNHL (24). Slow blood flow in the inner ear may cause stasis and, subsequently, hypoxia-induced hair cell damage; however, timely treatment can improve microcirculation to obtain a better outcome. Hearing threshold is affected by hair cell damage and reflects hearing function and the therapeutic effect of treatment on the inner ear (25). The presence of tinnitus symptoms indicates that the lesion has reached the vestibular labyrinth, which increases the difficulty of treatment and rehabilitation. Serum CRP level is a common inflammatory response index, and changes in 
Table VI. Multivariate logistic regression analysis.

$95 \%$ C.I. for $\operatorname{EXP}(B)$

\begin{tabular}{lcccccrr}
\cline { 6 - 7 } Factors & $\mathrm{B}$ & S.E, & Wals & Sig. & Exp (B) & Lower & Upper \\
\hline Age & 0.040 & $0 .-47$ & 0.721 & 0.021 & 1.435 & 0.949 & 1.142 \\
Time of onset & -1.160 & 0.146 & 1.201 & 0.011 & 0.833 & 0.120 & 1.135 \\
Hearing threshold & 0.003 & 0.060 & 0.002 & 0.022 & 1.003 & 0.118 & 1.127 \\
Tinnitus & 0.778 & 1.070 & 0.530 & 0.001 & 1.078 & 1.033 & 17.727 \\
Plasma viscosity & -7.14 & 0.561 & 0.061 & 0.002 & 0.490 & 0.265 & 35.945 \\
Serum CRP level & 0.042 & 0.432 & 0.031 & 0.014 & 1.403 & 0.117 & 1.457 \\
\hline
\end{tabular}

$\mathrm{B}$ is the constant term. SE is the standard deviation. Wals is the chi-square value. Sig is the P-value. Exp (B) is the dominance ratio. $95 \%$ CI of $\operatorname{EXP}(\mathrm{B})$ is $95 \%$ confidence interval of the dominance ratio.

A

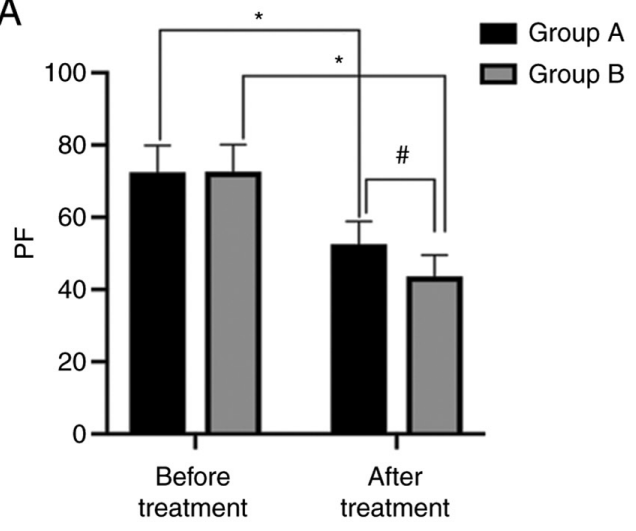

C

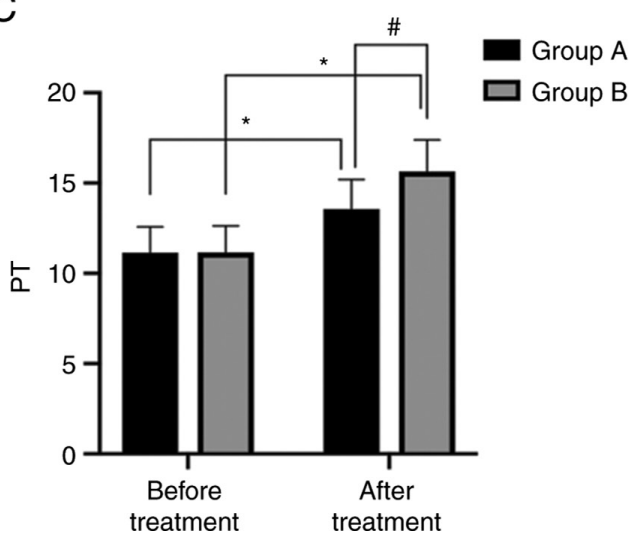

B

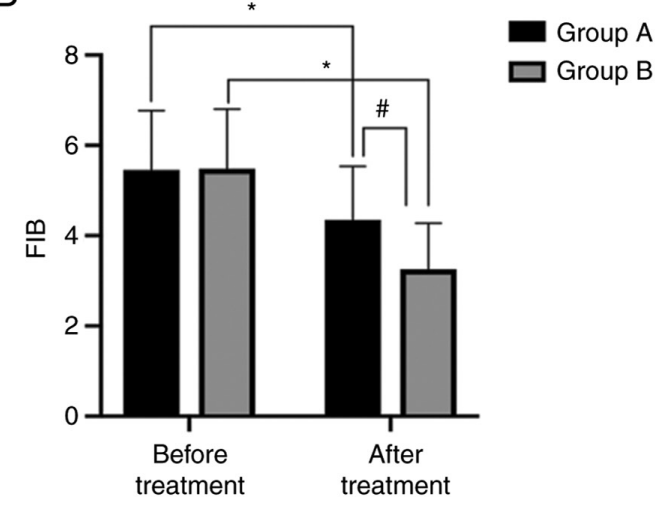

D

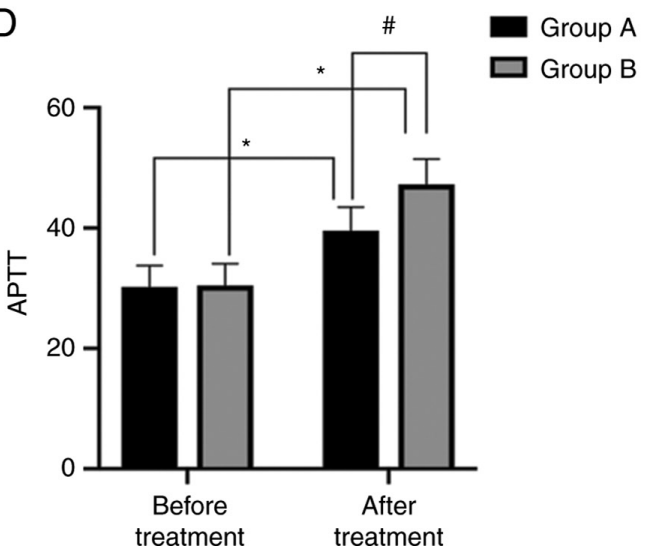

Figure 2. Comparison of parameters of coagulation function. (A) After treatment, PF levels in both groups significantly decreased, and were significantly lower in Group B than in Group A. (B) After treatment, FIB levels in both groups significantly decreased, and were significantly lower in Group B than in Group A. (C) After treatment, PT in both groups was significantly shortened, and was significantly shorter in Group B than in Group A. (D) After treatment, APTT in both groups was significantly shortened, and was significantly shorter in Group B than in Group A. " P $<0.05$ vs. pre-treatment; ${ }^{\#} \mathrm{P}<0.05$ vs. Group A. PF, platelet factor; FIB, fibrinogen; PT, prothrombin time; APTT, activated partial thrombin time.

its expression level affect the development and progression of SSNHL. High plasma viscosity indicates poor blood circulation. Therefore, monitoring plasma viscosity may be of great significance for efficacy evaluation of certain treatments (26). The aforementioned studies further verify the findings of the current study that the therapeutic effect of the treatment was poor if the corresponding pathological factors were present.
In summary, alprostadil combined with $\mathrm{HBO}$ was an effective treatment for SSNHL. However, certain shortcomings need to be explored in future studies. For example, the efficacy of SSNHL treatment is different among different hearing loss types and detailed analysis is yet to be completed. Additionally, the population size used in this study was relatively small. Other important influencing factors for the prognosis of SSNHL (such as the levels of serum lipids) will be examined in future studies. 


\section{Acknowledgements}

Not applicable.

\section{Funding}

No funding was received.

\section{Availability of data and materials}

The datasets used and/or analyzed during the current study are available from the corresponding author on reasonable request.

\section{Authors' contributions}

TF, QZ, JW, XW and YG conceived and designed the research and interpreted the results of experiments. QZ, JW and XW contributed to the design of the study and interpretation of experimental results. TF and YG performed experiments, analyzed data, prepared figures, and drafted the manuscript. TF and YG edited and revised the manuscript. All authors read and approved the final version of the manuscript.

\section{Ethics approval and consent to participate}

All patients and their families agreed to participate in this study and signed an informed consent form. This study was approved by the Medical Ethics Committee of Zibo Central Hospital.

\section{Patient consent for publication}

Not applicable.

\section{Competing interests}

The authors declare that they have no competing interests.

\section{References}

1. Furuhashi A, Matsuda K, Asahi K and Nakashima T: Sudden deafness: Long-term follow-up and recurrence. Clin Otolaryngol Allied Sci 27: 458-463, 2002.

2. Research on deafness. Doctoral dissertations. Am Ann Deaf 147: 230-233, 2002

3. Hirayama M, Shitara T, Okamoto M and Sano H: Idiopathic bilateral sensorineural hearing loss: Its clinical study in cases with rapidly progressed deafness. Acta Otolaryngol Suppl 534: 39-42, 1996.

4. Qiu L, Zheng X, Xie F, Zhang M, Zhang J, Yuan SB and Zeng GZ: Clinical observation on the different frequency hearing damages in sudden deafness treated by electroacupuncture combined with western medicine comprehensive therapy. World J Acupunct Moxibustion 22: 22-27, 2012.

5. Inagaki T, Cureoglu S, Morita N, Terao K, Sato T, Suzuki M and Paparella MM: Vestibular system changes in sudden deafness with and without vertigo: A human temporal bone study. Otol Neurotol 33: 1151-1155, 2012

6. Ersoy Callioglu E, Tuzuner A, Demirci S, Cengiz C and Caylan R Comparison of simultaneous systemic steroid and hyperbaric oxygen treatment versus only steroid in idiopathic sudden sensorineural hearing loss. Int J Clin Exp Med 8: 9876-9682, 2015.

7. Augustin AJ, Diehm C, Grieger F and Bentz J: Alprostadil infusion in patients with dry age related macular degeneration: A randomized controlled clinical trial. Expert Opin Investig Drugs 22: 803-812, 2013.
8. Güneysu E, Kocman AE, Özatik O, Ovali C, Can B, Alatas İÖ and Sevin MB: The effects of iloprost and alprostadil on ischemia-reperfusion injury in preventing inflammation, tissue degeneration, and apoptosis in rat skeletal muscle. Turk J Med Sci 47: 1028-1036, 2017.

9. de Pont AC, de Jonge E and Vroom MB: Hyperbaric oxygen for acute carbon monoxide poisoning. N Engl J Med 348: 557-560, 2003.

10. Li J, Shi Q, Wei Q and Han P: Re: Efficacy and safety of mirodenafil for patients with erectile dysfunction: A meta-analysis of three multicenter, randomized, double-blind, placebo-controlled clinical trials. Aging Male 18: 71, 2015.

11. Wei KU, Liu Y, Jing WU, Wang Q, Tong B and Duan M: Meta-analysis of the clinical efficacy of treatment of sudden deafness using hyperbaric oxygen combined with drug in China. Chin J Otol 12: 93-98, 2014.

12. Arndt S, Laszig R, Aschendorff A, Hassepass F, Beck R and Wesarg T: Cochlear implant treatment of patients with single-sided deafness or asymmetric hearing loss. German version. HNO 65: 586-598, 2017 (In German).

13. Weiss D, Böcker AJ, Koopmann M, Savvas E, Borowski M and Rudack C: Predictors of hearing recovery in patients with severe sudden sensorineural hearing loss. J Otolaryngol Head Neck Surg 46: 27, 2017.

14. Zhai S, Ning Y, Guiliang Z, Yuhua Z and He Q: Effect of retreatment on the end-stage sudden deafness. Cell Biochem Biophys 62: 403-406, 2012.

15. Merchant SN, Durand ML and Adams JC: Sudden deafness: Is it viral? ORL J Otorhinolaryngol Relat Spec 70: 52-62, 2008.

16. Ban JH, Hong SJ, Lee SY, Lee KC and Kim CG: A clinical analysis of psychogenic hearing loss presenting as sudden deafness. Korean J Otolaryngol Head Neck Surg 48: 572-576, 2005.

17. Domachevsky L, Keynan Y, Shupak A and Adir Y: Hyperbaric oxygen in the treatment of sudden deafness. Eur Arch Otorhinolaryngol 264: 951-953, 2007.

18. Kornberg A: Letter: Prostaglandin E1 therapy with alprostadil and risk reduction of early hepatic cellular carcinoma after liver transplantation-authors' reply. Aliment Pharmacol Ther 43: 173-174, 2016.

19. Liu Y, Sun D, Shao S, Jiang W, Sun Z and Li Z: The effect of hyperbaric oxygen therapy to different degree of hearing loss and types of threshold curve in sudden deafness patients. Lin Chung Er Bi Yan Hou Tou Jing Wai Ke Za Zhi 24: 890-894, 2010 (In Chinese).

20. Tatar S, Orhan N, Yilmaz CU, Arican N, Ahishali B, Kucuk M, Elmas I, Kaya M and Toklu AS: Hyperbaric oxygen therapy for five days increases blood-brain barrier permeability. Undersea Hyperb Med 44: 345-355, 2017.

21. Jiang J, Wang S and Tong K: Synergic effect of alprostadil injection and ginaton in treating sudden deafness. Lin Chung Er Bi Yan Hou Tou Jing Wai Ke Za Zhi 25: 597-599, 2011 (In Chinese).

22. Xin W, Wen HL, Zhao Q and Zhao ZW: Blood dilution, hyperbaric oxygen, anticoagulation treatment for acute carbon monoxide poisoning 115 patients. Chinese Journal of Medicinal Guide: 45-47, 2008.

23. Hernández-Tejedor A, Cabré-Pericas L, Martín-Delgado MC, Leal-Micharet AM and Algora-Weber A; EPIPUSE study group: Evolution and prognosis of long intensive care unit stay patients suffering a deterioration: A multicenter study. J Crit Care 30: 654.e1-e7, 2015.

24. Ren J and Lu Y: Sudden deafness in the elderly. Lin Chuang Er Bi Yan Hou Ke Za Zhi 12: 257-259, 1998 (In Chinese).

25. Liberman MC: Noise-induced hearing loss: Permanent versus temporary threshold shifts and the effects of hair cell versus neuronal degeneration. Adv Exp Med Biol 875: 1-7, 2016.

26. Gao D, Shao J, Jin W, Xia X and Qu Y: Correlations of serum cystatin $C$ and hs-CRP with vascular endothelial cell injury in patients with systemic lupus erythematosus. Panminerva Med 60: 151-155, 2018.

This work is licensed under a Creative Commons Attribution-NonCommercial-NoDerivatives 4.0 International (CC BY-NC-ND 4.0) License. 